\title{
Construction and characterisation of a modular microfluidic system: coupling magnetic capture and electrochemical detection.
}

5 Neus Godino ${ }^{1}$, Detlef Snakenborg ${ }^{2}$, Jörg P. Kutter ${ }^{2}$, Jenny Emnéus ${ }^{2}$, Mikkel Fougt Hansen ${ }^{2}$, F. Xavier Muñoz ${ }^{1}$, F. Javier del Campo ${ }^{1 *}$.

${ }^{1}$ Instituto de Microelectrónica de Barcelona, IMB-CNM (CSIC).

Esfera UAB. Campus Universitat Autónoma de Barcelona.

08193 - Bellaterra. Barcelona, Spain.

2 DTU Nanotech

Technical University of Denmark (DTU)

15 Department of Micro and Nanotechnology

Oersteds Plads

Building 345east, room no. 254

DK-2800 Kongens Lyngby, Denmark

20

To be submitted to: Microfluidics and nanofluidics

\begin{tabular}{|l|l|}
\hline Proofs to: & F. Javier Del Campo \\
\hline Phone: & SP -935947700 ext.:1306 \\
\hline Fax: & SP -935801496 \\
\hline Email: & Franciscojavier.delcampo@cnm.es \\
\hline
\end{tabular}

25

Version: 2.0

Date: $14-5-2009$ 


\section{Abstract}

This work presents the fabrication and characterisation of a versatile lab-on-a-chip system that combines magnetic capture and electrochemical detection. The system comprises a silicon chip featuring a series of microband electrodes, a PDMS gasket

5 that incorporates the microfluidic channels, and a polycarbonate base where permanent magnets are hosted; these parts are designed to fit so that wire bonding and encapsulation are avoided.

This system can perform bioassays over the surface of magnetic beads and uses only $50 \mu \mathrm{L}$ of bead suspension per assay. Following detection, captured beads are

10 released simply by sliding a thin iron plate between the magnets and the chip. Particles are captured upstream from the detector and we demonstrate how to take further advantage of the system fluidics to determine enzyme activities or concentrations, as flow velocity can be adjusted to the rate of the reactions under study. We used magnetic particles containing $\beta$-galactosidase and monitored the enzyme activity amperometrically by the oxidation of 4-aminophenol enzymatically produced from 4 -aminophenyl- $\beta$-D-galactopyraniside. The system is able to detect presence of enzyme down to approximately $50 \mathrm{ng} \mathrm{mL}^{-1}$.

Keywords: PDMS, channel electrode, microfluidics, aminophenol, galactosidase, lab on a chip, electrochemistry. 


\section{Introduction}

Magnetic particles and amperometry have been combined in the past for (bio)electroanalytical purposes (Choi et al., 2002, Do and Ahn, 2008, Farrell et al., 2004, Gabig-Ciminska et al., 2004, Goral et al., 2006, Llopis et al., 2009) and several literature reports describe systems where particles are directly immobilised on a channel wall opposite the electrodes (Choi et al., 2002, Do and Ahn, 2008). Choi et al. used interdigitated microelectrode structures operated under generatorcollector mode in an example close to our approach (Choi et al., 2002). More recently, a system was reported where the magnetic particles are elegantly immobilised by means of an array of NiFe permalloy blocks (Do and Ahn, 2008). However, we believe that the overall performance of those systems may improve if the magnetic particles are captured upstream from the electrodes and not opposite them. This modification would take full advantage of the system microfluidics, which provides controlled and well-defined mass transport conditions leading to higher steady state currents. Amperometry is a relatively simple but very important detection technique in lab-on-a-chip devices because currents recorded in channel electrodes (Alden et al., 1998, Morland and Compton, 1999) under laminar flow are directly proportional to analyte concentration (Amatore et al., 2008, Cooper and Compton, 1998, Rees and Compton, 2008, Rossier and Girault, 2001, Tokuda and

20 Matsuda, 1974). The main advantages of this system are its re-usability and that its configuration can be changed easily so the user can try several electrode configurations with the same holder. Moreover, because the functionalised beads are magnetically captured upstream from the electrodes, the user can adjust the fluid flow rate to the kinetic parameters of the chemical reactions under study to optimise detection. Later, the magnetic particles are released blocking the magnetic field. This is easy to achieve sliding a thin soft-iron piece between the magnets and the chip, and flushing a buffer solution through the system.

We are presenting a versatile lab-on-a-chip that can be used in a broad range of (bio)electroanalytical assays. It is also important to point out that this system does 30 not require wire bonding nor resin based encapsulation, thus eliminating two of the most important sources of cost in traditional microsystems. The system avoids these burdens thanks to its modular construction consisting of the following three main parts, which will be further described below: (i) a demountable rigid plastic body, (ii) a soft PDMS gasket that simultaneously provides the microfluidic channel and sealing and (iii) a silicon chip featuring a set of microelectrodes that are connected using spring-loaded pins. 
The present system has been designed to develop electrochemical immunoassays, (Rossier and Girault, 2001, Másson et al., 1995) and can perform two different operations: the capture of magnetic beads, including the mixing of different solutions, and the electrochemical detection of species present in solution. Note,

5 that there are variations to the way the system may be operated, particularly during the sample extraction and labelling stages, but broadly the system works as follows: after extracting an enzyme-labelled analyte from a sample using suitably functionalised magnetic particles, these are injected by a syringe pump into the capture chamber moulded in the PDMS gasket. There, the strong magnetic field

10 exerted by two permanent magnets captures and spreads them over the side of the chip upstream from the microelectrodes. Next, a solution containing a nonelectroactive enzyme substrate is pushed through the system, at a flow rate chosen to take advantage of the prevailing reaction kinetics. The electroactive enzymatic product is then detected downstream at the electrodes before the solution leaves 15 the system.

The incorporation of magnetic particles is an important aspect of this lab-on-a-chip device because of the flexibility they offer (Thomas et al., 2004). In contrast to most electrochemical biosensors, where the bio-recognition element is linked to the electrode surface, this system uses the surface of magnetic beads (Llopis et al., 20 2009, Verpoorte, 2003, Gijs, 2004) to host the biological reaction of interest. Using magnetic beads, the same electrodes may be used for the detection of different analytes and they may also last longer. On the other hand, thanks to the complete absence of wire bonds or encapsulating resin in the system, the chip can easily be replaced if the electrodes become irreversibly damaged.

25 We demonstrate the operation of our cell using streptavidin coated magnetic beads and a biotinylated $\beta$-galactosidase as model system. To enable electrochemical detection, we use 4-aminophenyl- $\beta$-D-galactopiranoside, PAPG, as the enzyme substrate because it yields p-aminophenol, PAP, a well known reversible electroactive species that is readily oxidised at a mild potential of $0.25 \mathrm{~V}$ vs.

$30 \mathrm{Ag} / \mathrm{AgCl}$ (Compton and Banks, 2007, Conant and Pratt, 1926, Plichon and Faure, 1973, Yamaguchi and Mitsugi, 1997, Niwa et al., 1993, Másson et al., 1995, Do and Ahn, 2008). We also use finite element methods to aid in the characterisation of the system and to interpret the enzyme kinetics from measured transient currents (Nicholson and Shain, 1964). 


\section{Experimental}

\subsection{System Fabrication and Characterisation}

\subsubsection{Holder fabrication}

The system holder consists of three parts: The top part was made in

5 polymethylmethacrylate (PMMA) and the bottom in polycarbonate (PC). The middle part was a polydimethylsiloxane (PDMS) inlay as shown in Figure 1. This PDMS part provided watertight sealing and contained all the microfluidic features of the system. Direct micromilling (Snakenborg et al., 2006) and casting were used to define the various geometric features of the three parts.

A)

PMMA top part:

1: Microchannels $\mathrm{d}=800 \mu \mathrm{m}$

2: PDMS gasket socket

3: Spring loaded pins socket

PDMS gasket:

4: Magnetic bead microchamber

5: Microchannel

6: Ridge

7: Cylindrical protusions

8: Ribs

Chip with the microelectrodes

PC bottom part:

9: chip socket

10: iron piece socket

11: magnet socket

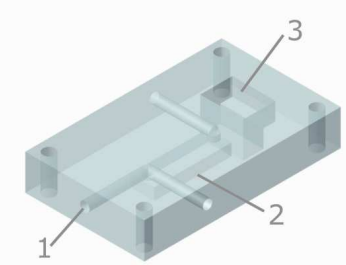

B) PDMS gasket

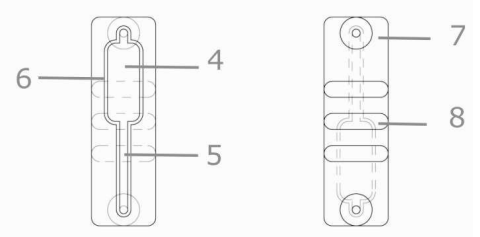

C)

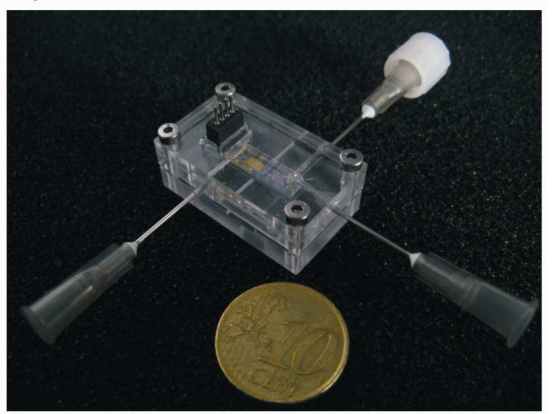

Figure $1 A$ ) $3 D$ Schematic representation of the system assembly (to scale). The top part is made in PMMA; a PDMS gasket contains the microfluidics and provides sealing against a chip featuring a set of microband electrodes; the chip base is made in PC. B) Three different perspectives of PDMS gasket: general, bottom and top C) Photograph of the system.

The top part features the fluidic interconnections and a pocket for the PDMS inlay. Short pieces of silicone tubing (about $5 \mathrm{~mm}$ long, $0.8 \mathrm{~mm}$ OD) were inserted in the channels to seal the fluidic connections water-tight. The solutions are fed in and out of the system through blunt syringe needles with an outer diameter slightly larger than the inner diameter of the silicon tubing. Thus, when the needles are inserted, they push the silicone tubing against the PMMA holder and a tight seal is achieved. 
The round cross-section of the channels in the PMMA substrate was achieved by structuring two PMMA sheets ( $3 \mathrm{~mm}$ thick) using a $0.8 \mathrm{~mm}$ ball-end milling tool and subsequent UV-assisted thermal bonding (Shinohara et al., 2007, Tsao et al., 2007). Finally, spring-loaded pins (Preci-dip, Switzerland) were locked into holes, drilled through both PMMA layers, to provide electrical connections to the chip.

The gasket was fabricated by micromiling a PMMA mould and subsequent casting of PDMS (Duffy et al., 1998, McDonald and Whitesides, 2002). It contained all microfluidic features, consisting of a cavity $2 \mathrm{~mm}$ wide, $150 \mu \mathrm{m}$ high and $5 \mathrm{~mm}$ long joined to a channel $500 \mu \mathrm{m}$ wide and of the same height that leads the solution out of the system after passing over the detector. The cell was designed so that the wide part of the channel sat on the chip directly above the permanent magnets where magnetic beads would be captured. Downstream, the length of the electrodes was controlled by the width of the $500 \mu \mathrm{m}$ exit channel. A series of special features moulded on the top and bottom faces of the PDMS piece improved the sealing. Three centred ribs $4 \mathrm{~mm}$ wide, $1 \mathrm{~mm}$ long and $150 \mu \mathrm{m}$ high were built on the top face to distribute the pressure evenly over the gasket, and a ridge 200 $\mu \mathrm{m}$ wide and $80 \mu \mathrm{m}$ high, protruding out the bottom side of the PDMS around the chamber and the microchannel, improved the sealing against the chip; these features reduced the required clamping force and thereby the risk of channel deformation.

The bottom part contains two pockets for housing the chip and two magnets as well as a notch that allows sliding a metal piece between the magnets and the chip. Two $\mathrm{Nd}-\mathrm{Fe}-\mathrm{B}$ permanent magnets (Lika, Denmark, $2 \times 2 \times 2 \mathrm{~mm}^{3}$ ) were inserted into the pocket, with opposite vertical polarization, to maximize the magnetic force on the beads in the channel (Smistrup et al., 2008). The purpose of the iron piece (500 $\mu \mathrm{m}$ thick) was to shield the chip from the magnetic field and hence release the beads held inside the microchannel. This allowed performing consecutive experiments without altering the geometric features of the system, and improved the reproducibility of the measurements.

30 The purpose behind using a PDMS inlay in combination with a rigid holder was to have a permanent piece of hardware that could perform different operations depending on the geometry of this PDMS gasket; in this particular case this meant magnetic bead capture followed by electrochemical detection. Other possibilities include filtration, ion exchange or solid phase extraction operations, to name three.

\section{$35 \quad$ 2.1.2 Chip design and fabrication.}

The electrode chip design consists of 4 microbands, 3 bands of $500 \mu \mathrm{m}$ width and 1 band of $1 \mathrm{~mm}$ width, separated by $100 \mu \mathrm{m}$ gaps. The wider band is intended for use 
as auxiliary electrode while the remaining three bands may be used as working or pseudo-reference electrode in any combination. However, to avoid potential shifts during measurements, the first band upstream was used as the reference electrode. The gold microbands were fabricated using standard photolithographic

5 techniques. A $50 \mathrm{~nm}$ adhesion layer of $\mathrm{Ti}$ and a $200 \mathrm{~nm}$ layer of Au were sequentially deposited by e-beam evaporation, and the electrodes were defined by lift-off.

\subsection{Chemicals and instrumentation}

$\mathrm{KCl}(99 \%), \quad \mathrm{KNO}_{3}(99 \%), \quad \mathrm{K}_{4} \mathrm{Fe}(\mathrm{CN})_{6} .3 \mathrm{H}_{2} \mathrm{O}$ (99\%), p-aminophenol, N,N-

10 Dimethylformamide, DMF (99.8\%, spectrophotometric grade) were purchased from Sigma-Aldrich. All chemicals were ACS analytical grade and were used as received without any further purification. Aqueous solutions were prepared using pure deionized water $(18 \mathrm{M} \Omega \cdot \mathrm{cm})$. Buffer $\mathrm{Z}$ was prepared using commercial PBS tablets (Invitrogen) reaching a $0.1 \mathrm{M}$ concentration and adding $1 \mathrm{mM} \mathrm{MgSO}_{4}$ and $20 \mathrm{mM}$ $\mathrm{KCl}$.

The magnetic beads were streptavidin coated Dynabead $\AA$ MyOne (Invitrogen), $1 \mu \mathrm{m}$ diameter $\left(3.14 \mu \mathrm{m}^{2}\right)$. The specific area of these beads is $2.2-3.8 \mathrm{~g} \mathrm{~m}^{-2}$, and their nominal binding capacity is $20 \mu \mathrm{g}$ protein per $\mathrm{mg}$ of beads. The interbead volume once inserted and immobilised on the system, assuming a monolayer of particles packed evenly over the surface of the reaction chamber, is approximately $0.48 \mathrm{~nL}$ (note that the total volume in the capture chamber is $150 \mathrm{~nL}$ ). All electrochemical measurements were performed using a three-electrode configuration and a CHI700C bipotentiostat ( $\mathrm{CH}$ Instruments, Texas, USA) connected to a PC. All electrodes were made from gold, including the pseudoreference electrode, and were integrated on the same silicon chip. The flow was controlled using a syringe pump, NE1000 (New Era Pump Systems, NY). The electrodes were occasionally passivated from prolonged operation in $\mathrm{p}$-aminophenol solutions. In those cases the electrodes were removed and cleaned by rinsing the chip in DMF (Goncalves et al., 2000), or directly replaced by new ones.

\subsection{Bead functionalization}

Magnetic beads were functionalized outside the device following a protocol described elsewhere (Baldrich and Muñoz, 2008). Briefly, a $40 \mu \mathrm{L}$ volume of streptavidin-coated beads $\left(10 \mathrm{mg} \cdot \mathrm{mL}^{-1}\right), 1 \mu \mathrm{m}$ in diameter, was first rinsed several times using buffer $Z$. Next, $150 \mu \mathrm{L}$ of buffer and $10 \mu \mathrm{L}$ of $1 \mathrm{mg} \cdot \mathrm{mL}^{-1}$ biotinylated $\beta$ galactosidase were added, and the solution was stirred for 30 minutes at room 
temperature. The excess enzyme physisorbed on the beads was removed in a new wash. The functionalized magnetic beads solution was diluted to a concentration of $3.33 \mathrm{mg} \cdot \mathrm{mL}^{-1}$.

\subsection{Operation of the system}

5 The working principle of our system is as follows: first, a given volume of functionalized beads containing the labelled target is captured inside the microfluidic holder by permanent magnets. Then, a solution containing the substrate is introduced in the channel and the product of the enzyme reaction is electrochemically detected downstream using a microband electrode under controlled-flow conditions. The capture of the target and the necessary incubations are physically separated from the electrochemical detector. The magnetics beads are easily released by blocking the magnetic field with a thin iron slab between the magnets and the chip. This allows carrying out several measurements sequentially without changing any geometrical features and increasing reproducibility.

\section{Theory}

The sequence of operation in the system can be divided in three steps: (i) magnetic bead capture, (ii) enzymatic reaction and (iii) amperometric detection. This section describes the simulation of the detection, as the principles underpinning movement and capture of magnetic beads can be found elsewhere (Lund-Olesen et al., 2007, Mikkelsen et al., 2005, Smistrup et al., 2008).

Fluid flow, diffusional mass transport, and enzyme and electrochemical reactions were considered in a two-dimensional domain, as depicted in Figure 2 . The reaction between 4-aminophenyl- $\beta$-D-galactopyranoside (PAPGP) and $\beta$-galactosidase $(\beta G A L)$ produces $p$-aminophenol (PAP) as summarized in the following scheme:

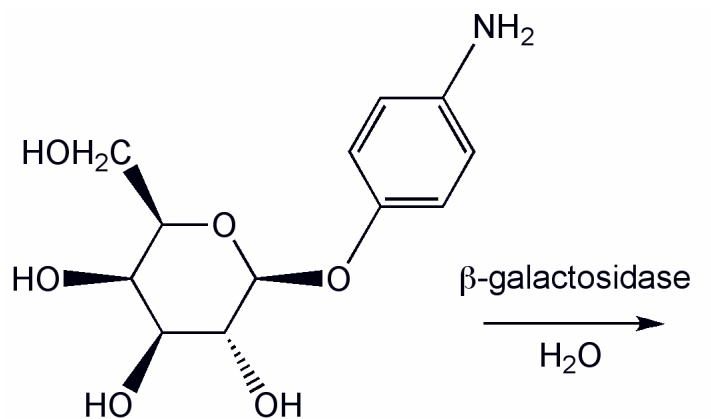

PAPGP<smiles>Nc1ccc(O)cc1</smiles>

galactose p-aminophenol

Reaction scheme 1 
A Michaelis-Menten type mechanism (Voet and G.Voet, 1995) was assumed to govern the kinetics of this reaction:

$$
E+S \boxminus \underset{k_{-1}}{k} E S \stackrel{k_{c a t}}{\longrightarrow} P+E
$$

5 Therefore, the activity of the $\beta$-galactosidase immobilized on the beads was described by:

$$
\frac{\partial[S]}{\partial t}=-k_{c a t}\left[E_{\beta G A L}\right] \frac{[S]}{K_{M}+[S]}
$$

where $[S]$ is the concentration of PAPG, $\left[E_{\beta G A L}\right]$ is the enzyme concentration linked to the beads, and $k_{c a t}$ is the turn over number in $\mathrm{s}^{-1}$ and $K_{M}$ is the Michaelis Menten constant in mol $\mathrm{L}^{-1}$. For the sake of simplicity, the behaviour of the captured magnetic beads was represented in our model by a boundary, (Figure 2) so that equation (2) was defined as a boundary mass flux.

The macroscopic fluid flow was described by the steady-state Navier-Stokes equations or an incompressible Newtonian fluid

$$
\begin{gathered}
\rho(\vec{v} \cdot \nabla) \vec{v}-\eta \nabla^{2} \vec{v}+\nabla p=0 \\
\nabla \cdot \vec{v}=0
\end{gathered}
$$

where $\rho$ and $\eta$ are the density and the viscosity of the fluid, $\vec{v}$ is the velocity vector and $p$ is the pressure. The mass transport of species within the channel was defined by convection and diffusion

$$
\frac{\partial c_{i}}{\partial t}=D_{i} \nabla^{2} c_{i}-\vec{v} \cdot \nabla c_{i}
$$

where $c_{i}$ is the concentration and $D_{i}$ is the diffusion coefficient of each species involved.

At the electrode, we considered the two-electron oxidation of PAP to piminoquinone (PIQ) as:

$\mathrm{E}$

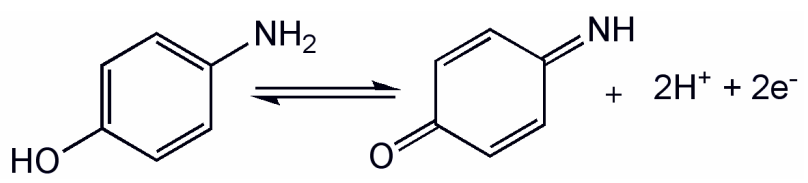

Reaction scheme 2 .

We assumed infinitely fast electrode kinetics such that the current was limited by diffusion (Bard and Faulkner, 2001) and described by the following integral:

$$
I=\left.n F D_{P A P} w \int_{0}^{x} \frac{\partial[P A P]}{\partial y}\right|_{y=0} d x
$$


where $D_{P A P}$ is the diffusion coefficient of PAP, [PAP] is the concentration of $\mathrm{p}$ aminophenol, $n$ is the number of exchanged electrons, $F$ is the Faraday constant, $w$ is the width, and $x_{e}$ is the length of the microband electrode.

The above system of equations was solved for the geometry outlined in Figure 2 using COMSOL Multiphysics 3.4 (COMSOL, SE) running on a Windows based PC.

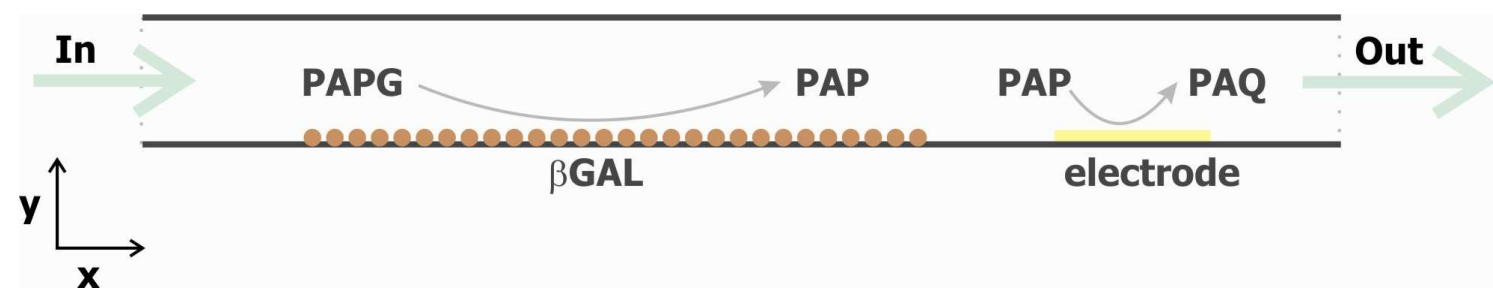

Figure 2. Schematic representation of the model. 2-dimensional section of the microchannel (not to scale). The enzyme activity over the surface of immobilised beads is considered as a flux boundary condition.

\section{Results and Discussion}

\subsection{Electrochemical detection}

The mass transport rates inside the cell were investigated by cyclic voltammetry using equimolar solutions of ferro/ferricyanide. Figure $3 \mathrm{~A}$ shows typical cyclic voltammograms (CVs) obtained at $50 \mathrm{mVs}^{-1}$ for flow rates of 2 and $10 \mu \mathrm{L} \mathrm{min}{ }^{-1}$, respectively. The fact that the voltammetric waves are centred around $\mathrm{OV}$ is due to the potential of the gold pseudo-reference employed.

The electrodes were always electrochemically activated prior to any measurements. This activation step consisted in the application of a series of 10 s potential pulses, alternating between $-1 \mathrm{~V}$ and $\mathrm{OV}$ in a $0.5 \mathrm{M} \mathrm{KNO}_{3}$ electrolyte solution.

The current measured by a microband band inside a microfluidic channel is described by (Compton et al., 1993):

$$
I=0.925 n F c w\left(x_{e} D\right)^{2 / 3}\left(\frac{Q}{h^{2} d}\right)^{1 / 3}
$$

where $h$ is half of the height of the microchannel and $Q$ is the (volumetric) flow rate. $\mathrm{C}$ is the concentration of the electroactive species and, for ferro/ferricyanide, $D$ is $6.5 \cdot 10^{-10} \mathrm{~m}^{2} \mathrm{~s}^{-1}$ (Bard and Faulkner, 2001).

One of the most important features of the system is its simplicity and reproducibility, so that the microfluidic cell is easily opened and closed to clean or replace the chip inside it. According to equation (7), channel geometry has a direct effect on the faradaic current, so the PDMS gasket was specially designed to minimise variability in channel height due to compression of the PDMS when the 
system is closed. This particular design facilitated an even distribution of the pressure over the entire channel and kept the height variation over the channel within a narrow tolerance.

We thoroughly tested our system by repeatedly opening and closing the cell; we 5 used different gaskets, and recorded CVs at a range of fluid flow rates. Figure 3B shows that the system behaved in agreement with equation (7) within the chosen range of flow rates. The current residual standard deviation (RSD) in ferrocyanide value was systematically better than (below) $3 \%$. Steady state currents, measured in ferro/ferricyanide solutions, were used in combination with equation (7) to calibrate the actual geometry of the channel prior to further studies. Figure 3B also shows the theoretical steady state currents according to equation (7) for a microchannel of height $105 \pm 5 \mu \mathrm{m}$. This $5 \mu \mathrm{m}$ interval corresponds to a $3 \%$ change in the measured current only, and we relied on this approach to calibrate our system.

A)

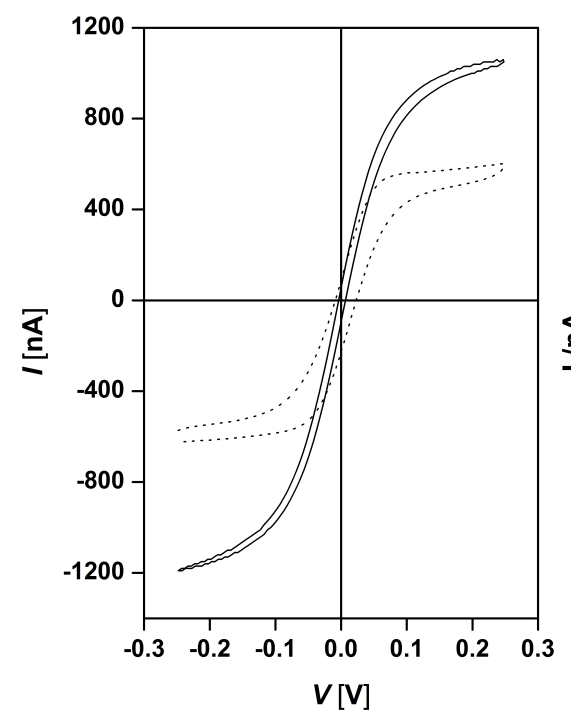

B)

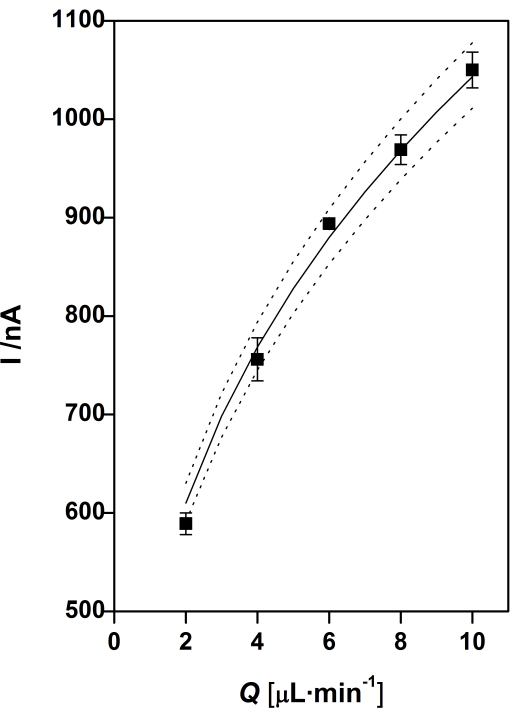

15 Figure 3. A) Cyclic voltammograms for $1 \mathrm{mM}$ equimolar solutions of ferro/ferricyanide at $50 \mathrm{mVs}^{-1}$ at $2(\cdots)$ and $10 \mu \mathrm{L} \mathrm{min} \mathrm{m}^{-1}(-)$; the potential was measured vs. a Au pseudo-reference electrode. B) Steady state currents for $1 \mathrm{mM}$ equimolar solutions of ferro/ferricyanide at $50 \mathrm{mVs}^{-1}$ for different flow rates: experimental ( $\mathbf{0})$, theoretical response for a $105 \mu \mathrm{m}$ channel height (-) and for deviations of $\pm 5 \mu \mathrm{m}(\cdots)$ error bars based on 6 measurements. RSD is below $3 \%$.

In addition, cyclic voltammetry of known ferro/ferricyanide concentrations was also used to check the working condition of the system between experiments. 


\subsection{Study of enzyme activity: simulations explain system behaviour}

An important objective of this work was to demonstrate the suitability of this detection system for electrochemical immunoassays. To demonstrate the operation of the system, we present an enzyme activity study under different flow rate conditions. We analysed the relation between enzyme activity, flow rate and measured current. In addition to the experimental work, we used numerical modelling to improve our understanding of the system behaviour.

In the experiments, $50 \mu \mathrm{L}$ of a bead suspension was injected at $50 \mu \mathrm{L} \cdot \mathrm{min}^{-1}$ and

10 trapped by the magnets. The substrate PAPG was then introduced through the channel with immobilized beads at a lower flow rate $\left(0.1-10 \mu \mathrm{L} \cdot \mathrm{min}^{-1}\right)$ to minimise bead loss during the operation. The rate of enzyme product generation was controlled by the flow rate of the substrate solution. Thus, although a high flow rate might be beneficial from the viewpoint of the electrochemical detection, it would not leave enough time to generate a measurable amount of enzyme product, and therefore a smaller current would be detected. Conversely, at lower flow rates, the enzyme will be able to turn more substrate into product, leading to an increased current. Figure 4, shows this for a $1: 480$ bead dilution measured at $0.25 \mathrm{~V}$ vs. Au at different flow rates. The flow rate was held constant at a certain value for some time and then it was swiftly changed to a (much) higher or lower value, which resulted the appearance of current spikes. The injected substrate concentration was $1 \mathrm{mM}$ to ensure its excess compared to the enzyme concentration and working at $V_{\max }$ throughout the experiment.

Simulations were used to interpret and predict the behaviour of the system. In our case, several physico-chemical phenomena were occurring simultaneously and, despite the approximations, simulations helped in the understanding of how these phenomena related to each other. Note that there are two different channel widths in the PDMS gasket; $2 \mathrm{~mm}$ in the magnetic bead capture chamber and $500 \mu \mathrm{m}$ along the detection channel. This means that the symmetry of the real geometry cannot be accounted for by our 2D model, and a 3-dimensional model would be needed to model the geometry more accurately. Our approximation was to assume the same width both for the capture chamber and the detector channel (500 $\mu \mathrm{m})$ and then multiplying it by a geometrical correction factor, $K_{g}=16$, to the enzyme kinetic constant. We used kinetic values obtained from an independent experiment at microdisk electrode arrays and simulated the enzyme kinetics of $\beta$-galactosidase according to a Michaelis-Menten mechanism. The resulting values were a $K_{m}$ of $3.15 \cdot 10^{-4} \mathrm{~mol} \cdot \mathrm{L}^{-1}$ and a $k_{\text {cat }}$ of $0.18 \mathrm{~s}$ (equation (2)). The channel height was 
estimated electrochemically as described in section $4.1(105 \mu \mathrm{m})$. The diffusion coefficient values for PAPG and PAP were estimated by the Wilke-Chang method (Wilke and Chang, 1955), which yields values accurate to within $10 \%$ or better for small molecules. These values were $3.6 \cdot 10^{-10} \mathrm{~m}^{2} \cdot \mathrm{s}^{-1}$ for PAPG and $8.6 \cdot 10^{-10} \mathrm{~m}^{2} \cdot \mathrm{s}^{-1}$

5 for $\mathrm{p}$-aminophenol. The enzyme surface concentration for $1: 480$ dilution is $8.6 \cdot 10^{-8}$ $\mathrm{mol} \cdot \mathrm{m}^{-2}$, which was estimated from an initial concentration of magnetic beads at $10^{10}$ beads $\cdot \mathrm{mL}^{-1}$; an injected volume of $50 \mu \mathrm{L}$; enzyme concentration on the beads of $0.8 \mathrm{mg} \cdot \mathrm{mL}^{-1}$ (see supplementary information); and the maximum area that these injected beads could occupy.

10 The experimental sequence was simulated and Figure 4 shows the agreement between the experimental and the simulated transient currents. As expected, the current increased inversely with the flow rate due to the longer residence time of the substrate in the bead capture zone containing the enzyme. Note the positive and negative current peaks appearing when the flow rate is switched from a higher to a lower speed and vice versa. These peaks were explained considering that a product concentration corresponding to the previous flow rate was displaced at a different flow rate over the electrode, and hence influenced the current as predicted by equation (7). If we for instance consider the switch from $0.1 \mu \mathrm{l} \cdot \mathrm{min}^{-1}$ to $1 \mu \mathrm{L} \cdot \mathrm{min}^{-1}$ ((d) to (c) in Fig. 4), there was a higher product concentration in the system at $0.1 \mu \mathrm{L} \cdot \mathrm{min}^{-1}$ because the substrate and the immobilised enzyme had more time to react. Then, the flow rate was increased and the solution containing this concentration of product was suddenly pushed at $1 \mu \mathrm{L} \cdot \mathrm{min}^{-1}$ and, as expected from equation (7), the recorded current increased. Once all the solution containing the higher product concentration was flushed over the electrode, a new steady state current of lower magnitude was achieved. Figure 4 shows that although there was very good agreement between the experimental and simulated steady state currents, the peaks were somewhat different. The simulated peaks were in general higher and sharper than the experimental ones. This is explained if we take into account the fact that the experimental system was driven by a syringe pump, 30 where the switch from one flow rate to another was not immediate, and that stabilisation of the new conditions was delayed. The simulation, on the other hand, did not consider these non-idealities and abruptly changed from one flow rate to the next. However, what matters is the remarkable agreement between the experimental and the simulated steady-state currents, as shown in Figure 4.

35 Experimental measurements were repeated several times to demonstrate the reproducibility of the system. In each measurement, a new aliquot of magnetic beads was introduced, measured and released. Moreover, control of the flow rate in this system allows the study of chemical kinetics in general, but also of enzyme 
kinetics. Kinetic parameters can be estimated with our system because the measured currents reflect the competition between transport of the reagents and the rate of the chemical reaction under study. Our system can work under a very wide range of flow rates, which allows both access to kinetic parameters of different magnitudes and the detection of very small amounts of electroactive material, as will be shown below.

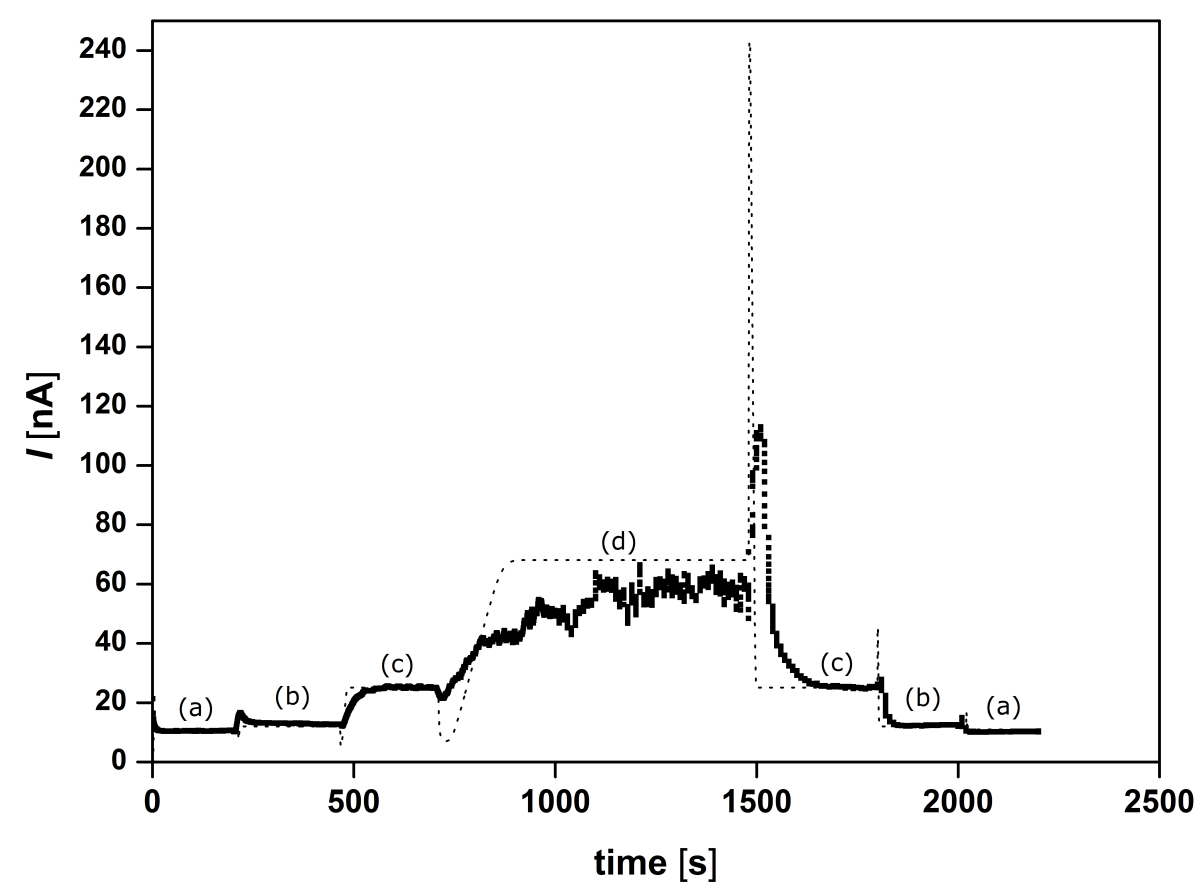

Figure 4. (ロ) Experimental current at 0.25V vs. Au pseudo-reference and different flow rates for $50 \mu \mathrm{L}$ of a $1: 480$ beads dilution captured in the system and a substrate concentration of $1 \mathrm{mM}$. (a) $10 \mu \mathrm{L} \cdot \mathrm{min}^{-1}$, (b) $5 \mu \mathrm{L} \cdot \mathrm{min}^{-1}$. (c) $1 \mu \mathrm{L} \cdot \mathrm{min}^{-1}$ and (d) $0.1 \mu \mathrm{L} \cdot \mathrm{min}^{-1}$. The dotted line corresponds to the simulated current under the same conditions.

Table 1. Experimental and simulated steady state currents at different flow rates as depicted in Figure 4. The experimental error is reported as the standard deviation of three independent measurements

\begin{tabular}{|l|l|l|l|}
\hline & $\mathrm{I}_{\text {experimental }}(\mathrm{nA})$ & $\mathrm{RSD} / \%$ & $\mathrm{I}$ modeling $(\mathrm{nA})$ \\
\hline $0.1 \mu \mathrm{L} \cdot \mathrm{min}^{-1}$ & $60.1 \pm 3$ & 5.0 & 68.1 \\
\hline $1 \mu \mathrm{L} \cdot \mathrm{min}^{-1}$ & $26.0 \pm 1.6$ & 6.2 & 25.1 \\
\hline $5 \mu \mathrm{L} \cdot \mathrm{min}^{-1}$ & $12.1 \pm 0.9$ & 7.5 & 12.0 \\
\hline $10 \mu \mathrm{L} \cdot \mathrm{min}^{-1}$ & $9.3 \pm 1.0$ & 10.8 & 11.0 \\
\hline
\end{tabular}




\subsection{Detection of $\beta$-galactosidase by pulsed or stopped flow}

The appearance of peaks during the transition between flow rates (Figure 4) served as the basis for an operation mode that increases the sensitivity of the system. This is similar to the technique described by Karlberg in (Francis et al., 2002), or Merkoçi et al. in (Llopis et al., 2009), and it is of paramount importance in biosensors, and more specifically in immunoassays, aiming at the detection of very small concentrations down to $\mathrm{ng} \cdot \mathrm{L}^{-1}$ or even $\mathrm{pg} \cdot \mathrm{L}^{-1}$. Figure 4 shows the current peaks for the different flow rate transitions. The height of the positive peaks was several times larger than the preceding steady state current, which represents a substantial signal enhancement.

To exploit this feature, rather than measure under a continuous flow of substrate, we operated under stopped-flow conditions for a short period of time. During this time the reaction proceeded under stagnant conditions and its rate was controlled by diffusion of the substrate to the bead surface. Next, the pump was switched on and this volume, containing the electroactive PAP, was pushed over the working microband electrode and a current peak was observed. Simulations were able to model these events and Figure 5iError! No se encuentra el origen de la referencia. shows a series of simulated peaks obtained after different incubation times.

20 The model was solved using the same conditions as those from Figure 4. However, to model the pulsed flow behaviour, the solution was left to react for a certain time and then pushed at $5 \mu \mathrm{l} \cdot \mathrm{min}^{-1}$. Figure 5 shows how these peaks increase with incubation time, and it is interesting to note that the peak currents seem to reach a plateau at longer incubation times. This is because after long incubation periods, the immobilised enzyme consumes most of the substrate present in the solution. This may be a problem because diffusion of the product into the detector channel may be detrimental to sensitivity. To avoid problems like this, incubation times must be carefully watched.

Another interesting feature is the duration of the simulated flow pulses, which was always less than 5s. This may explain the different shape and height of the experimental peaks because our syringe pump took longer than this to reach the desired flow rates.

Figure 6 shows the experimental results for 1:480 and 1:960 dilutions at different incubation times. The experimental peaks were considerably lower than the simulated ones. This difference increased with incubation times as the concentration of product also increased. However, the current ratio between both dilutions was constant for every incubation time, which was an excellent indication 
of a consistent and reproducible bead capture. The fact that the current peak for the 1:480 dilution was twice as high as the peak at 1:960 dilution implied that the enzyme concentration also doubled due to the amount of captured beads. Every measurement was repeated at least three times and the results were highly

5 reproducible. This also suggested that the captured beads were very stable and that very few beads were lost during the measurements.

We can conclude that this operational mode leads to outstanding sensitivity enhancements. For example, in the case of a $1: 480$ dilution at $0.1 \mu \mathrm{L} \cdot \mathrm{min}^{-1}$ the steady-state current was around 60nA (see Figure 4). However, after only 10 10 minutes of incubation the signal increased six times, up to around 400nA for the same flow rate. Therefore, using stopped-flow operation may be very beneficial for cases where the biological target is present in the sample at extremely low concentrations. During testing, it was possible to detect presence of enzyme products in a $1: 10^{4}$ dilution of magnetic beads, equivalent to approximately $50 \mathrm{ng}$ $15 \mathrm{~mL}^{-1}$.

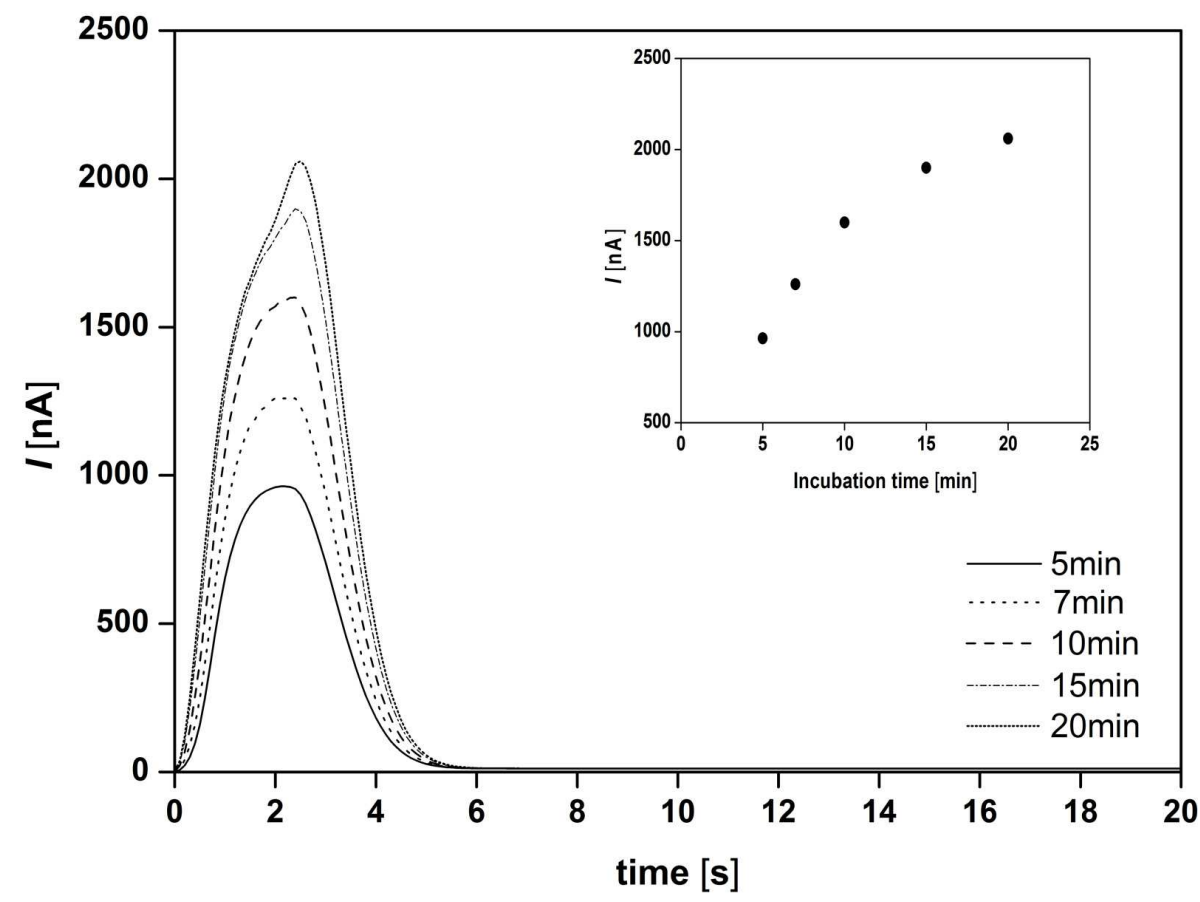

Figure 5 Simulated peaks after different incubation times: 5, 7, 10, 15 and 20 minutes. Following incubation the solution was pushed at $5 \mu \mathrm{L} \cdot \mathrm{min}^{-1}$ for a 1:480 beads dilution capture. 


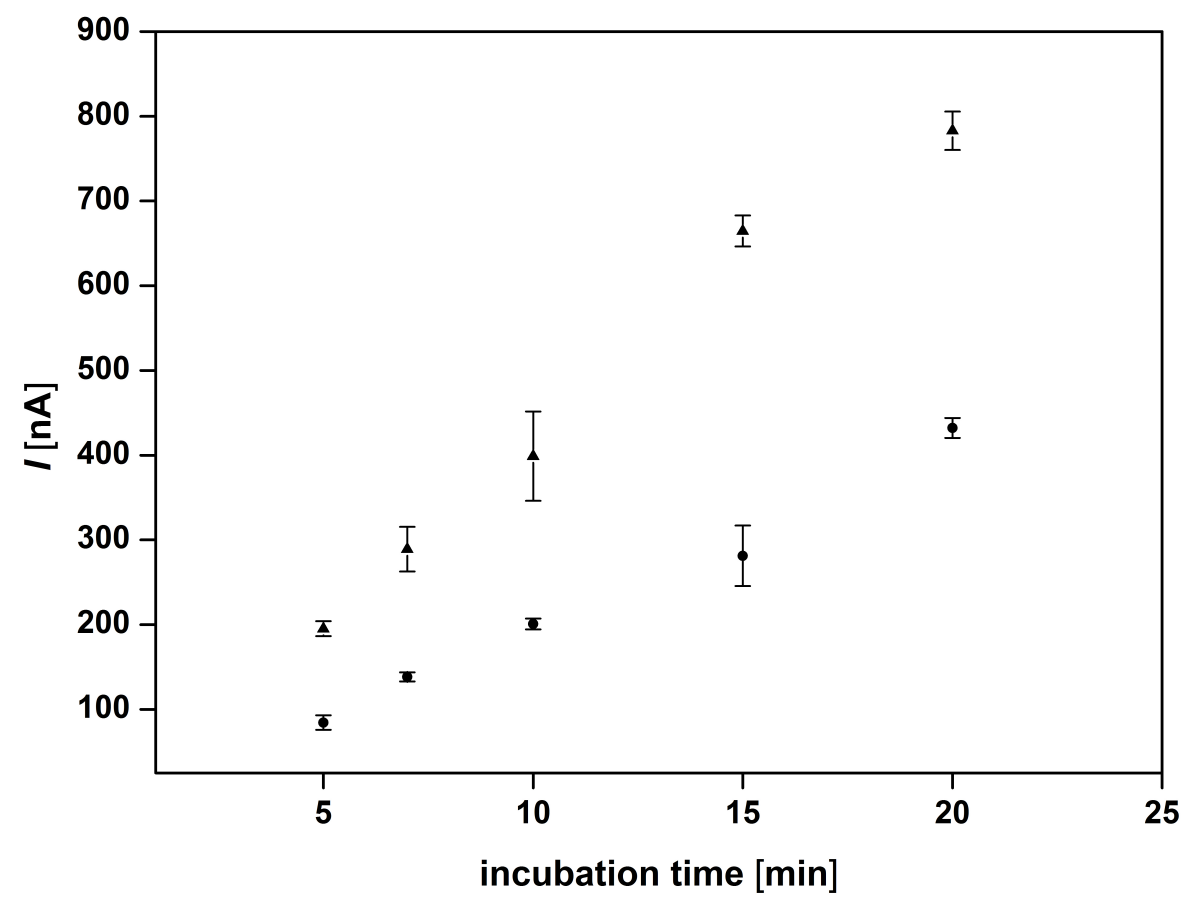

Figure 6. Comparison of experimental current peaks for particles captured from two different magnetic bead dilutions: 1:480(山) and 1:960 (,) (The initial concentration of magnetic beads is $10 \mathrm{mg} \cdot \mathrm{mL}^{-1}$. The measurements were done at $0.25 \mathrm{~V} \mathrm{vs}$. Au 5 pseudo-reference at different incubation times. Solution was pushed at $5 \mu \mathrm{L} \cdot \mathrm{min}^{-1}$ after the incubation.

\section{Conclusions}

Lab-on-a-chip devices are understood as an engineering challenge involving integration of new processes, new materials and geometry scaling. From this point of view, we aimed to produce an easy-to-use and reusable device. We focused this work to improve the control over the chemical or biological reactions taking place during bioassays by adjusting flow rate. This is what we have presented here: a very versatile lab on a chip system that integrates magnetic capture and electrochemical detection in a microfluidic environment and where flow can be

15 adjusted depending on the chemical kinetics of the system to optimize the detection. The system versatility stems from its highly modular nature, where both the PDMS embedded microfluidics and the chip-based microelectrode set can be readily replaced without need for wire bonding or encapsulation. The PDMS gasket incorporates a $150 \mu \mathrm{m}$ high channel containing two distinct regions: a chamber

$202 \mathrm{~mm}$ wide where the magnetic beads can be trapped by a permanent magnetic field, and a $500 \mu \mathrm{m}$ microchannel, leading directly to the set of microband electrodes where enzyme reaction products are detected. Such microfluidic features 
can be easily changed replacing this PDMS gasket for a new one of different channel configuration. This allows the final user to adapt the whole system to new experimental needs. Moreover, once the detection has been carried out and the particles are no longer needed, they can be easily released by blocking the

5 magnetic field by sliding a thin iron slab between the magnets and the chip.

Although the system presented here was designed to immobilise magnetic particles upstream from the working electrode in electrochemical immunoassays, it could also be used more generically in other electroanalytical applications. We have used the well-known $\beta$-galactosidase and PAPGP enzyme and substrate system, and we have been able to determine the enzyme surface concentration over our magnetic beads (see supporting material). Thus, we have demonstrated its operation and possible applicability to the field of bioassays and we conclude that the system could be used in any enzyme-based bioassay (e.g., displacement or sandwich immunoassays based on conventional ELISA). Time of analysis and/or sensitivity could be further improved with the use of interdigitated microband structures operated in generator-collector mode, or by extending the incubation time of the substrate in the presence of immobilised enzyme-modified magnetic particles when the system is operated in stopped-flow mode.

Finite element simulations were of great help in understanding the system behaviour. Our simulations suggested that the system flow suffers some delay before it reaches the desired velocity. In spite of this, the system affords high levels of reproducibility and sensitivity, which makes it an attractive tool for the development of novel electrochemical bioassays. In addition to immunoassays, the system can also be used in comparative studies of enzyme activities towards different substrates, or in different media, or to compare the activity of different enzymes on a given substrate.

\section{Acknowledgements}

The authors would like to acknowledge funding from the Spanish ministry of Science and Innovation through the Microbactometer project. NG is supported by CSIC's JAE program and JdC is supported by a Ramón y Cajal fellowship. 


\section{References}

Alden, J. A., Feldman, M. A., Hill, E., Prieto, F., Oyama, M., Coles, B. A., Compton, R. G., Dobson, P. J. \& Leigh, P. A. (1998) Channel Microband Electrode Arrays for Mechanistic Electrochemistry. Two-Dimensional Voltammetry: Transport-Limited Currents. Anal. Chem., 70, 1707-1720.

Amatore, C., Da Mota, N., Sella, C. \& Thouin, L. (2008) General concept of highperformance amperometric detector for microfluidic (bio)analytical chips. Anal. Chem., 80, 4976-4985.

Baldrich, E. \& Muñoz, F. X. (2008) Enzyme shadowing: Using antibody-enzyme dually-labeled magnetic particles for fast bacterial detection. Analyst, 133, 1009-1012.

Bard, A. J. \& Faulkner, L. R. (2001) Electrochemical methods: Fundamentals and Applications, Chichester, Wiley.

Choi, J. W., Oh, K. W., Thomas, J. H., Heineman, W. R., Halsall, H. B., Nevin, J. H., Helmicki, A. J., Henderson, H. T. \& Ahn, C. H. (2002) An integrated microfluidic biochemical detection system for protein analysis with magnetic bead-based sampling capabilities. Lab. Chip., 2, 27-30.

Compton, R. G. \& Banks, C. E. (2007) Understanding Voltammetry, Singapore, World Scientific Publishing.

20 Compton, R. G., Fisher, A. C., Wellington, R. G., Dobson, P. J. \& Leigh, P. A. (1993) Hydrodynamic Voltammetry with Microelectrodes. Channel Microband Electrodes: Theory and Experiment. J. Phys. Chem., 97, 10410-10415.

Conant, J. B. \& Pratt, M. F. (1926) The irreversible oxidation of organic compounds I. The oxidation of aminophenols by reagents of definite potential. JACS, 48, 3178-3192.

Cooper, J. A. \& Compton, R. G. (1998) Channel Electrodes - A Review. Electroanalysis, 10, 141-155.

Do, J. \& Ahn, C. H. (2008) A polymer lab-on-a-chip for magnetic immunoassay with on-chip sampling and detection capabilities. Lab. Chip., 8, 542-549.

Duffy, D. C., Mcdonald, J. C., Schueller, O. J. A. \& Whitesides, G. M. (1998) Rapid prototyping of microfluidic systems in poly(dimethylsiloxane). Anal. Chem., 70, 4974-4984.

Farrell, S., Ronkainen-Matsuno, N. J., Halsall, H. B. \& Heineman, W. R. (2004) Bead-based immunoassays with microelectrode detection. Anal. Bioanal. Chem., 379, 358-367.

Francis, P. S., Lewis, S. W., Lim, K. F., Carlsson, K. \& Karlberg, B. (2002) Flow analysis based on a pulsed flow of the solution: theory, instrumentation and applications. Talanta, 58, 1029-1042.

Gabig-Ciminska, M., Holmgren, A., Andresen, H., Bundvig Barken, K., Wümpelmann, M., Albers, J., Hintsche, R., Breitenstein, A., Neubauer, P., Los, M., Czyz, A., Wegrzyn, G., Silfversparre, G., Jürgen, B., Schweder, T. \& 
Enfors, S. O. (2004) Electric chips for rapid detection and quantification of nucleic acids. Biosens. Bioelectron., 19, 537-546.

Gijs, M. A. M. (2004) Magnetic bead handling on-chip: New opportunities for analytical applications. Microfluid. Nanofluid., 1, 22-40.

5 Goncalves, D., Faria, R. C., Yonashiro, M. \& Bulhoes, L. O. S. (2000) Electrochemical oxidation of o-aminophenol in aqueous acidic medium: Formation of film and soluble products. J. Electroanal. Chem., 487, 90-99.

Goral, V. N., Zaytseva, N. V. \& Baeumner, A. J. (2006) Electrochemical microfluidic biosensor for the detection of nucleic acid sequences. Lab. Chip., 6, 414421.

Llopis, X., Pumera, M., Alegret, S. \& Merkoçi, A. (2009) Lab-on-a-chip for ultrasensitive detection of carbofuran by enzymatic inhibition with replacement of enzyme using magnetic beads. Lab. Chip., 9, 213-218.

Lund-Olesen, T., Bruus, H. \& Hansen, M. F. (2007) Quantitative characterization of magnetic separators: Comparison of systems with and without integrated microfluidic mixers. Biomed. Microdevices, 9, 195-205.

Másson, M., Liu, Z., Haruyama, T., Kobatake, E., Ikariyama, Y. \& Aizawa, M. (1995) Immunosensing with amperometric detection, using galactosidase as label and p-aminophenyl-B-D-galactopyranoside as substrate. Anal. Chim. Acta, 304, 353-359.

Mcdonald, J. C. \& Whitesides, G. M. (2002) Poly(dimethylsiloxane) as a material for fabricating microfluidic devices. Acc. Chem. Res., 35, 491-499.

Mikkelsen, C., Fougt Hansen, M. \& Bruus, H. (2005) Theoretical comparison of magnetic and hydrodynamic interactions between magnetically tagged particles in microfluidic systems. J. Magn. Magn. Mater., 293, 578-583.

Morland, P. D. \& Compton, R. G. (1999) Heterogeneous and Homogeneous EC and ECE Processes at Channel Electrodes: Analytical Wave Shape Theory. J. Phys. Chem. B, 103, 8951-8959.

Nicholson, R. S. \& Shain, I. (1964) Theory of stationary electrode polarography single scan and cyclic methods applied to reversible, irreversible, and kinetic systems. Anal. Chem., 36, 706-723.

Niwa, O., Xu, Y., Brian Halsall, H. \& Heineman, W. R. (1993) Small-volume voltammetric detection of 4-aminophenol with interdigitated array electrodes and its application to electrochemical enzyme immunoassay. Anal. Chem., $65,1559-1563$.

Plichon, V. \& Faure, G. (1973) Thin-Layer Electrochemistry - Applications to Chemical-Kinetics - Example of Para-Aminophenol. J. Electroanal. Chem., 44, 275-290.

Rees, N. V. \& Compton, R. G. (2008) Hydrodynamic microelectrode voltammetry. Russ. J. Electrochem., 44, 368-389.

Rossier, J. S. \& Girault, H. H. (2001) Enzyme linked immunosorbent assay on a microchip with electrochemical detection. Lab. Chip., 1, 153-157. 
Shinohara, H., Mizuno, J. \& Shoji, S. (2007) Low-temperature direct bonding of poly(methyl methacrylate) for polymer microchips. IEEJ Transactions on Electrical and Electronic Engineering, 2, 301-306.

5

Smistrup, K., Bu, M., Wolff, A., Bruus, H. \& Hansen, M. F. (2008) Theoretical analysis of a new, efficient microfluidic magnetic bead separator based on magnetic structures on multiple length scales. Microfluid. Nanofluid., 4, 565573.

Snakenborg, D., Perozziello, G., Klank, H., Geschke, O. \& Kutter, J. P. (2006) Direct milling and casting of polymer-based optical waveguides for improved transparency in the visible range. Journal of Micromechanics and Microengineering, 16, 375-381.

Thomas, J. H., Kim, S. K., Hesketh, P. J., Halsall, H. B. \& Heineman, W. R. (2004) Microbead-based electrochemical immunoassay with interdigitated array electrodes. Anal. Biochem., 328, 113-122.

Tokuda, K. \& Matsuda, H. (1974) Theory of stationary current-voltage curves of redox-electrode reactions in hydrodynamic voltammetry. IX. Double electrodes in channel flow. J. Electroanal. Chem., 52, 421-431.

Tsao, C. W., Hromada, L., Liu, J., Kumar, P. \& Devoe, D. L. (2007) Low temperature bonding of PMMA and $\mathrm{COC}$ microfluidic substrates using UV/ozone surface treatment. Lab. Chip., 7, 499-505.

Verpoorte, E. (2003) Beads and chips: new recipes for analysis. Lab. Chip., 3, 60N.

Voet, D. \& G.Voet, J. (1995) Biochemistry, John Wiley \& Sons.

Wilke, C. R. \& Chang, P. (1955) A. I. Ch. E. Journal, 1, 264.

Yamaguchi, S. \& Mitsugi, S. (1997) Sensitive amperometry of 4-aminophenol (4AP) based on the catalytic current produced by the 4AP-diphorase-NADH system at a glassy carbon electrode and its application to enzyme assay. Effect of the inhibition of diaphorase by NADH. Anal. Sci., 13, 307-309. 